\title{
Para além dos discursos: o poder da afetividade*
}

\section{Miriam Adelman **}

Este livro, publicado 21 anos após o primeiro trabalho da autora $^{1}$, que ficou muito conhecido, demonstra o fecundo desenvolvimento tanto do pensamento desta estudiosa, quanto do feminismo acadêmico - que ao longo desse período muito cresceram com a procura de novos diálogos e a aquisição de novos interlocutores.

$\mathrm{O}$ argumento central do primeiro livro de Chodorow, assim como as diversas formas em que foi interpretado e criticado, ocupa um lugar importante na história da teoria feminista contemporânea. A tese exposta nesse livro, de que as estruturas e os papéis de maternagem ${ }^{2}$ dentro da família nuclear moderna são a base de estruturas diferentes de personalidade para homens $e$ mulheres - desenvolvendo nas meninas, através da relação com a mãe, uma capacidade relacional que contrasta com o eu mais autocentrado e fechado nos meninos, emergido da "exigência

\footnotetext{
* Resenha de Chodorow, Nancy. The Power of Feelings: Personal Meaning in Psychoanalysis, Gender and Culture. New Haven/London, Yale University Press, 1999. Recebida para publicação em setembro de 2003.

** Professora da Universidade Federal do Paraná e co-coordenadora, Núcleo de Estudos de Gênero/UFPR, Curitiba-PR, Brasil.miriamad@brturbo.com

1 The Reproduction of Mothering: Psychoanalysis and the Sociology of Gender. Berkeley, University of California Press, 1978. [A Psicanálise da Maternidade. uma Crítica a Freud a partir da Mulher. Rio de Janeiro, Rosa dos Tempos, 1978.]

2 O termo mothering traz à tona a idéia dos cuidados maternais, que incluem os referentes às necessidades físicas básicas de uma criança, mas enfatizam, ainda, os aspectos afetivos e emocionais, tão bem expressos em outro termo da língua inglesa, nurturing.
} 
Para além dos discursos

edípica" da separação - incentivou ampla discussão teórica e pesquisa empírica nos anos que seguiram. ${ }^{3}$

$\mathrm{Na}$ sua obra mais recente, Chodorow não pretende rejeitar sua tese anterior; porém, a forma em que trabalha com identidades de gênero e a noção do Eu e/ou da pessoa mostra algumas diferenças significativas, que em seguida ficarão evidentes. Seus interlocutores teóricos vêm de vários campos: dialoga claramente com a teoria psicanalítica clássica $e$ contemporânea e participa de debates que emergem da prática clínica psicanalítica, mas conversa também com a teoria feminista - em particular, com a vertente pós-moderna e sua noção de identidades fluídas ou fragmentadas - $e$, de forma particularmente eloqüente $e$ instigante, com algumas vertentes contemporâneas da antropologia cultural. Seu objetivo, como ela assinala logo de início, é

contribuir para nossa compreensão da subjetividade individual $[e]$ argüir em prol da existência de uma esfera não redutível de vida psíquica na qual constroem-se os significados pessoais e não conscientes. (p.1, tradução livre)

Chodorow empreende discussões em três campos principais. Em primeiro lugar, ela tenta mostrar os limites das perspectivas psico-desenvolvimentistas - provenientes do campo

${ }^{3}$ Bordo discute a forma em que este livro de Chodorow, assim como o da psicóloga feminista Carol Gilligan, se tornaram alvo de muita crítica, como obras que reificam ou essencializam uma diferença das mulheres. No entanto, o afinco empregado para se distanciar de uma alegada universalização de experiências de mulheres "brancas e de classe média" levou à desconsideração daquilo que talvez tenha sido o grande mérito do trabalho de ambas autoras (Bordo faz esta afirmação especificamente em relação à segunda): a forma pela qual o uso heurístico de um conceito de alteridade permite valorizar qualidades relacionais que podem ou não pertencer às mulheres culturalmente subordinadas - $e$, desta forma, produzir uma crítica cultural. BORDO, Susan. A feminista como o Outro. Revista Estudos Feministas, vol. 8, no 1, 2000; GilligAN, Carol. In a Different Voice: Psychological Theory and Women's Development. Cambridge, Harvard University Press, 1982. 
da psicanálise, da psicologia ou da psicologia social -, que concebem o processo de construção ou "desenvolvimento" do indivíduo como uma passagem por etapas ou fases estáveis e necessárias. Critica os conceitos de "tarefas específicas" fixas para cada fase do ciclo da vida (infância, adolescência, vida adulta, maturidade, etc.) e noções de formação de aspectos da identidade de gênero que corresponderiam a tais fases; contrapõe a este tipo de visão, uma perspectiva que, embora ela não identifique com a pós-moderna, deixa entrever algumas dívidas com a mesma. No entanto, neste ponto, ela apela à contribuição da teoria psicanalítica contemporânea e à forma pela qual esta emprega o conceito de transferência. Tal perspectiva, segundo ela, abandona a noção de uma estrutura psíquica relativamente consolidada $e$ fixa a partir dos processos relacionais da infância, para colocar nova ênfase nas "reconstruções" do passado no presente $e$ nos desdobramentos da dinâmica contínua da situação analítica. Assim, as pessoas não seriam mais concebidas como sendo determinadas pelo seu passado; o passado deixa de ter o caráter de fatos e processos consumados, e estabelece-se uma continuidade temporal na medida em que o passado se reconstrói no encontro terapêutico $e$ se atualiza nas interpretações $e$ relacionamentos que são feitos e refeitos, tanto nesse encontro quanto noutros espaços da vida cotidiana. Esta posição, segundo a autora, também faz parte de uma mudança que ocorre na filosofia psicanalítica da existência humana: transformou-se profundamente, desde a idéia clássica de Freud, que a concebe como determinada pela constante busca da satisfação das pulsões, para uma noção que a enxerga basicamente como "a busca da conexão (attachment)... a busca da construção de significados em relação ao Eu e ao Outros". ${ }^{4}$

\footnotetext{
${ }^{4}$ Neste sentido, sua visão conserva um vínculo estreito com o trabalho de outras teóricas da psicanálise feministas como Jessica Benjamin [The Bonds of Love: Psychoanalysis, Feminism and the Problem of Domination. New York, Pantheon Books, 1988] e da escola de feminist relational psychology, como as autoras que colaboraram no famoso volume Women's Ways of Knowing. BELENKY, M.F.,
} 
Para além dos discursos

Um segundo campo, ao qual recorre para dialogar e propor uma visão diferente, situa-se de frente para a literatura pósmoderna e a noção da "construção discursiva" da cultura e do Eu. Ao retomar a literatura específica sobre identidades de gênero, argumenta que a discursividade social, entendida também como um locus de relações de poder, pode fazer emergir a masculinidade e a feminilidade, mas não explica a relação de cada sujeito com as mesmas. Apropria-se da distinção feita por Irene Fast, que cunhou os conceitos de gênero objetivo e subjetivo, para fazer uma distinção entre padrões sociais e o que há de particular no modo pelo qual cada um/a elabora seu "sentido de gênero". Nesse aspecto, aparentemente, ela vai em direção contrária àquilo que enfatizava no seu primeiro livro - ou seja, a determinação sócio-cultural, na primeira infância, dos caminhos pelos quais a identidade de gênero será conduzida (embora também, poder-seia argumentar, que o conceito de "gênero objetivo" incorpora o conceito dos padrões culturais). O problema, para ela, é que equiparar a produção do gênero aos processos de "produção discursiva", caros às atuais teorias de gênero, pode conduzir a uma espécie de sobre-determinação, onde se produz, de fato, a morte do sujeito. Ela adverte, e voltará a discutir um pouco mais tarde, que essa perspectiva perde de vista a dimensão na qual os processos pessoais $e$ intersubjetivos - as fantasias $e$ desejos inconscientes que pertencem à história irrepetível de cada um/a, à história única de seus relacionamentos presentes e passados -

Clinchy, B.M., GoldBerger, N.R., \& TARULE, J.M. Women's Ways of Knowing: the Development of Self, Voice and Mind. New York, Basic Books, 1986.

${ }^{5}$ Ela comenta: "I have found particularly useful Fast's distinction... between 'objective' (I prefer her alternate term, 'observed') and subjective gender. Objective gender refers to observed differences in features of psychic or mental life or aspects of personality, character or behavior that tend to differentiate or characterize the sexes... subjective gender refers to personal constructions of masculinity and femininity - elements consciously or unconsciously linked to the sense of self as gendered". CHODOROW, N. The Power of Feelings... Op. cit., pp.103-104. 
Miriam Adelman

modificam, às vezes profundamente, os conteúdos da discursividade social. ${ }^{6}$ Nas últimas décadas, a crítica da literatura psicanalítica deixa claro que

Não há nenhuma feminilidade ou masculinidade genérica derivada de um fato cognitivo unívoco de gênero ou identidade de gênero, como conceito derivado da genitalidade e estabelecido ou no segundo ano de vida ou no período edípico. (p.124)

Assim, por válido que seja o conceito de gênero como cultural $e$ politicamente estabelecido - ou "discursivamente construído" -, sem a consideração de uma dimensão psicodinâmica que precisa ser captada sempre como processo singular, não há como apreender a complexa problemática da construção das identidades subjetivas. ${ }^{7}$

Finalmente, ela migra para outro campo do saber. Embora seja socióloga de formação, a autora afirma encontrar menos inspiração na sociologia - disciplina mais identificada com a busca de elementos "macro-sociais" e ainda fortemente marcada pela herança de concepções racionalistas do indivíduo - do que na antropologia cultural, cujo projeto central ela define como "o estudo dos significados culturais". (p.130)

Chodorow se interessa pela reconstrução de laços entre a psicanálise - definida neste momento como "antes de mais nada, uma teoria da construção do significado pessoal" (p.129) - e a antropologia cultural. Se, por um lado, segundo ela, a psicanálise

${ }^{6}$ Infelizmente, ela não incorpora a discussão da experiência transgênero - que fornece fortes evidências de formas singulares de encontro entre "discursividade social" e processos psicodinâmicos individuais - para subsidiar seu argumento neste ponto.

7 Talvez valha a pena citar mais um trecho para esclarecer o argumento da autora: "Any gender related category (man, woman, father, sister, brother, masculinity, femininity) gains meaning not just from language but from personally experienced emotion and fantasy in relation to a person connected to that label." (p.115) 
Para além dos discursos

se mostra deficiente na compreensão da complexidade sóciocultural do mundo que os indivíduos habitam, a antropologia - e, argumenta, mesmo a antropologia que toma por objeto específico as emoções e a afetividade - peca pela negação de uma dimensão psicodinâmica que permitiria enxergar a singularidade da pessoa. Mas uma "antropologia psicanalítica" - projeto em construção ao qual os esforços dela se somam ${ }^{8}$ - busca superar os determinismos de ambos tipos, contribuindo para validar as noções de construção pessoal de significados, mesmo para culturas onde noções "ocidentais" de indivíduo ou sujeito, aparentemente, não fariam sentido.

De fato, esta é a parte mais instigante do seu livro, que inicia com uma discussão da longa e complexa história de relação entre antropologia e psicanálise nas suas múltiplas vertentes. Assinala também que, a partir dos anos 70 ou 80, uma série de desenvolvimentos no campo das ciências sociais obrigaram a abertura de novos diálogos ${ }^{9}$ entre correntes de pensamento que, até então, pareciam ser ignoradas com certa facilidade, resultando, entre outros, no aparecimento de uma nova anthropology of self and feeling, "um campo auto-definido e autonomeado que mantém explicitamente um determinismo cultural $e$

8 "Psychoanalytic anthropology has developed more extensively than any other field, including pschoanalysis, a fine-tuned account of the complex intertwining of personal and cultural meaning, while remaining cognizant of both the intrapsychic and the cultural. Throughout the history of anthropology a great many studies... have described passion, feeling, internal conflict, uncertainty and interpersonal struggle, personal interpretations of cultural fantasies, stories and life history accounts, and the personal costs of cultural patterns and expectations - all documenting if not theorizing about individual psychodynamic personhood in different cultures." (p.131-132)

9 Entre estes, foi fundamental o surgimento do pós-estruturalismo, que põe em discussão muitas premissas caras à tradição nas ciências sociais. Embora este não seja o momento para aprofundar a questão, devo deixar claro que - como mencionei acima - Chodorow também se coloca a uma distância crítica do pensamento pós-estruturalista, incluindo Michel Foucault e sua noção da "construção discursiva" do sujeito. 
Miriam Adelman

anti-psicológico". (p.143) Neste sentido, a autora discute a obra de três antropólogos contemporâneos por ela considerados exemplares desta nova abordagem: Clifford Geertz, Michelle Rosaldo e Catherine Lutz. Para estes autores, a análise da cultura requer, por definição, a análise da construção das emoções na constituição da pessoa e os três se destacam pela forma brilhante com que demonstram a operação da cultura e da língua em tais processos. No entanto, segundo Chodorow, eles perpetuam uma falha crítica, reduzindo as emoções a "situated communication":

não há vida psicológica (ou pelo menos, nenhuma que seja de interesse para o estudioso da cultura) para além das categorias culturais consensuais e lingüisticamente rotuladas que servem para descrevê-la. (p.149)

No entanto, o trabalho etnográfico desses antropólogos dá vazão, alega Chodorow (em plena consonância com as conhecidas premissas da psicanálise sobre o "retorno" do que se encontra submerso ou denegado em qualquer discurso), à dimensão que eles tentam desenfatizar, fornecendo, assim, evidências da "fusão inseparável" de significados culturais e pessoais. ${ }^{10}$

Em seguida, através de um exame do trabalho etnográfico da antropóloga norte-americana Jean Briggs com os Inuit, do trabalho sobre sonhos de Waud Kracke com os índios Kagwahiv do Brasil, e as memórias da escritora marroquina Fátima Mernissi, entre outros, Chodorow busca subsídios para seu próprio

${ }^{10}$ Em referência específica à obra de Geertz, Chodorow argumenta "As different cultures name particular emotions, those cultures shape and give meaning to experience and make particular experiences more likely than others. What is needed in the investigation of these cultural meanings however is not a formal 'vacant sketch'... of coordinates, style and other cultural constructs but the ability to understand from the native's point of view how any particular enactment of a cultural form fuses such a sketch with the immediate, disruptive, intense and substantive... Cultural meaning and personal meaning are imposed only in the mind of the ethnographer." (p.148) 
Para além dos discursos

argumento: a produção cultural ou discursiva não esgota a produção da subjetividade ${ }^{11}$, há uma dimensão psicodinâmica que emerge de forma singular na vida de cada pessoa, sem a qual nenhuma noção de agency faria sentido. Recorre também ao fascinante trabalho de Obeyesekere, budista de Sri Lanka e estudioso da psicanálise. Segundo Obeyesekere, numa primeira fase, os conceitos desenvolvidos por Freud dos sistemas do inconsciente, pré-consciente e consciente poderiam ter (ou teriam) uma certa universalidade (à diferença de noções estruturalistas posteriores e de conceitos da vertente da "psicologia do ego", que exprimem processos culturalmente restritos); existe uma grande variação na forma e grau com que as culturas reconhecem os processos inconscientes e viabilizam sua expressão psicológica. (pp.182-183) Estas discussões me parecem bastante pertinentes também para os atuais debates na sociologia sobre a questão dos diversos Outros da modernidade, e sugerem, mais uma vez, que a noção corrente do sujeito como produto exclusivo da cultura ocidental moderna, deve, pelo menos em parte, ser reflexo de uma falta de compreensão desses Outros. ${ }^{12}$

A leitura deste livro pode ser de grande interesse para todos/as que trabalham com questões de identidade, cultura $e$ subjetividade. Com certeza, pertence ao corpo da literatura que busca complexificar a forma de entender os processos de construção do "sujeito" e das identidades de gênero, nesse sentido, representa também uma superação do primeiro livro da autora (qualificando, muito mais do que "invalidando", os argumentos da primeira obra). Por outro lado, é importante assinalar que o trabalho contribui para a compreensão de problemas metodológicos fundamentais da teoria social contemporânea, que vão além dos interesses específicos dos

${ }^{11}$ A psicanalista brasileira Maria Rita Kehl também elabora um argumento interessante neste sentido. KEHL, Maria Rita. A mínima diferença: masculino e feminino na cultura. Rio de Janeiro, Imago, 1996.

${ }^{12} \mathrm{O}$ excelente filme Samsara, do diretor indiano Pan Nalin, que tive a sorte de assistir em pré-estréia, também reforçou minha inquietação neste sentido. 


\section{Miriam Adelman}

estudos de gênero. Assim, mostra mais uma vez a viabilidade e a riqueza do pensamento feminista: a ousadia de sua voz crítica e a genialidade de sua forma particular de perceber o que muitos discursos recalcam. 